\title{
Adrenerge Urtikaria*
}

\section{Adrenergic Urticaria}

\author{
Autor \\ D. Kleinhans \\ Institut \\ Klinik für Dermatologie und Allergologie Stuttgart-Bad Cannstatt \\ (Ärztlicher Direktor: Prof. Dr. Peter von den Driesch)
}

\section{Bibliografie}

Dol $10.1055 / \mathrm{s}-2006-945033$

Akt Dermatol 2006; 32;

519-522 @ Georg Thieme

Verlag KG Stuttgart · New York

ISSN 0340-2541

Korrespondenzadresse

Prof. Dr. med.

Dieter Kleinhans

Kolpingstraße 2

70736 Fellbach

d.i.kleinhans@t-online.de

\section{Zusammenfassung \\ $\nabla$}

Die adrenerge Urtikaria ist nach eigener Erfahrung nicht so selten, wie es die wenigen publizierten Fälle vermuten lassen könnten. In einem Übersichtsreferat wird das Krankheitsbild anhand von fünf Fällen mit Fotos demonstriert und besprochen. Emotionaler Stress löst akute Schü-

Die Adrenergen Urtikaria wurde in ihrer Sonderstellung vom amerikanischen DermatologenEhepaar Shelley erkannt und 1985 in der Zeitschrift „The Lancet“ publiziert [1]; also nicht in einer dermatologischen Zeitschrift. Das war wohl der Grund dafür, dass das Krankheitsbild nur sehr langsam bekannt wurde. Im Titel ihrer Publikation formulieren Shelley u. Shelley [1] die von ihnen angenommene Ursache: „Adrenergic Urticaria: a new form of stress-induced hives".

In der Zusammenfassung beschreiben Shelley u. Shelley [1] die Hautveränderungen so: „Ausgedehnte urtikarielle Papeln von ein bis zwei Stunden Dauer; jede von einem auffallenden weißen Hof umgeben; entwickeln sich bei Stress, sind ein neuer Typ einer vom autonomen Nervensystem gesteuerten Urtikaria, kontrastierend zur cholinergen Urtikaria“. Im Gegensatz zur cholinergischen Urtikaria ist die Einzeleffloreszenz der adrenergen Urtikaria also ein zentraler roter Fleck, oft leicht erhaben, gelegentlich mit einer angedeutet urtikariellen Note, mit einem umgebenden hellen Hof.

Die adrenerge Urtikaria wird inzwischen in vielen Lehrbüchern genannt, nicht in allen. Sie scheint wenig bekannt zu sein. In der dermatologischen Literatur spielt die adrenerge Urtikaria kaum eine Rolle. Bis heute sind in medizinischen

* Publikationsfassung eines Vortrags beim „Stuttgarter Allergologie Update 2006“, April 2006 be aus, aber auch im Intervall sieht man meist einige wenige Effloreszenzen. Die betroffenen Patienten sind in der Regel ASS-intolerant in der Weise, dass sie auf Azetylsalizylsäure mit einem akuten makulös-urtikariellen Exanthem reagieren. Pathophysiologisch könnten stressbegleitende Neuropeptide oder auch Leukotriene eine Rolle spielen.

Datenbanken neben der Erstbeschreibung von Shelley u. Shelley [1] nur vier weitere Publikationen, genauer Fallbeschreibungen, gelistet [2-5]. Ein länger zurückliegendes Übersichtsreferat erscheint dabei nicht, weil die betreffende Zeitschrift in den Datenbanken nicht geführt wird [6].

\section{Klinisches Bild \\ $\nabla$}

Das klinische Bild der adrenergen Urtikaria soll anhand von fünf Fällen gezeigt und besprochen werden. Die Effloreszenzen sind in den einzelnen Fällen unterschiedlich lokalisiert. In den meisten Fällen sind es mäßig zahlreiche oder auch nur wenige Effloreszenzen. Akute Schübe führen zu exanthematisch ausgebreiteten Hautveränderungen. Krankheitstypisch sind kleine rote Flecke, z.T. leicht erhaben, mit hellem Hof (৫ Abb. 1, $\odot$ Abb. 2). Markiert man in einem Hautareal alle vorhandenen Effloreszenzen, sieht man, dass sie nach 1-2 Stunden verschwunden sind. In dieser Zeit entstehen in dem betreffenden Bereich neue Effloreszenzen. Ohne Markierung müsste man den Eindruck haben, es handele sich um persistierende Hauterscheinungen. In der $\odot$ Abb. 3 werden die typischen Hautveränderungen der adrenergen Urtikaria an den Oberschenkelrückseiten wegen der vorhandenen Erythrocyanose besonders deutlich. 


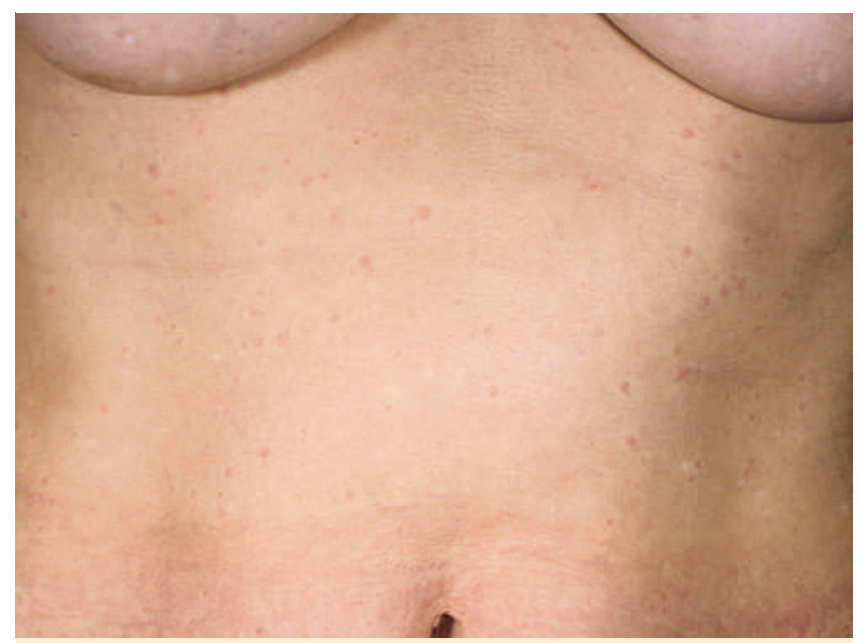

Abb. 139 Jahre alte Patientin, vorderer Stamm, typische rote Flecke, mit hellem Hof.

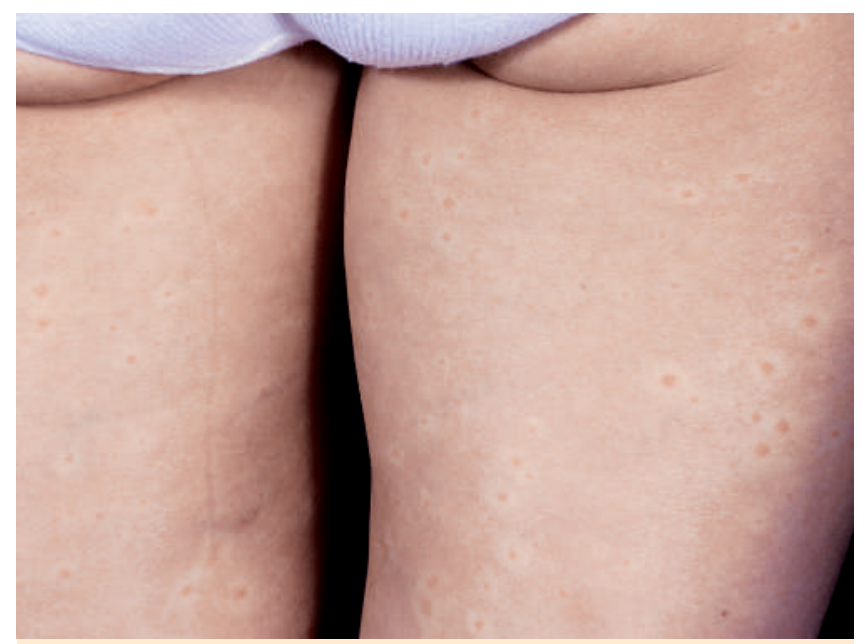

Abb. 345 Jahre alte Patientin, Oberschenkel, krankheitstypischer Befund.

Einen akuten exanthematischen Schub der adrenergen Urtikaria zeigt die Abb. 4: zahlreiche sehr kleine rote Flecke, ohne erkennbaren hellen Hof. Letzteres ist für akute Schübe einigermaßen typisch. Zur Diagnosestellung benötigt man in solchen Fällen zwei Hilfen: einmal das Markieren von Effloreszenzen, mit der Verlaufsbeobachtung; weiter die Inspektion anderer Hautpartien: In der Regel findet man in einem solchen Fall z.B. an den Unterarmen einige ältere krankheitstypischere Effloreszenzen. Ein akuter Schub entwickelt sich situationsabhängig, z.B. infolge Aufregung, Angst. Einen derartigen Schub sieht man in der Praxis eher selten.

Patienten mit adrenerger Urtikaria weisen in der Regel eine Azetylsalizylsäure-Intoleranz auf, wie das am Fall 5 gezeigt wird. Die

- Abb. 5 zeigt die adrenerge Urtikaria an einem Arm des Patienten; Abb. 6 die vordere Stammpartie desselben Patienten, ca. 45 Minuten nach der oralen Gabe von 100 mg Azetylsalizylsäure; mit einem ausgeprägten makulös-urtikariellen Exanthem.

Die Fotodokumentationen machen deutlich, dass das klinische Bild der adrenergen Urtikaria nicht so einheitlich ist, wie es die eingangs zitierte Beschreibung des Krankheitsbildes vermuten lassen könnte.

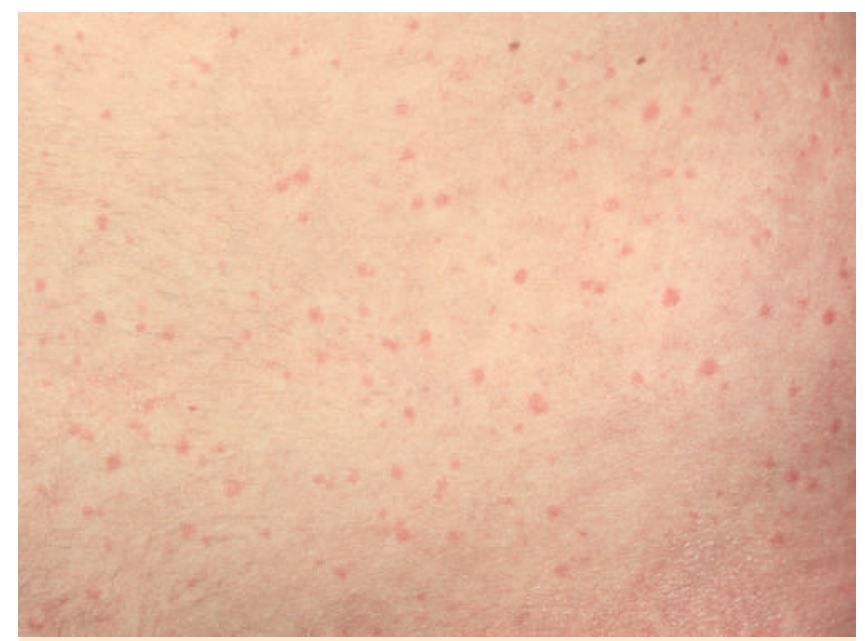

Abb. 235 Jahre alter Patient, krankheitstypisches Exanthem am vorderen Stamm.

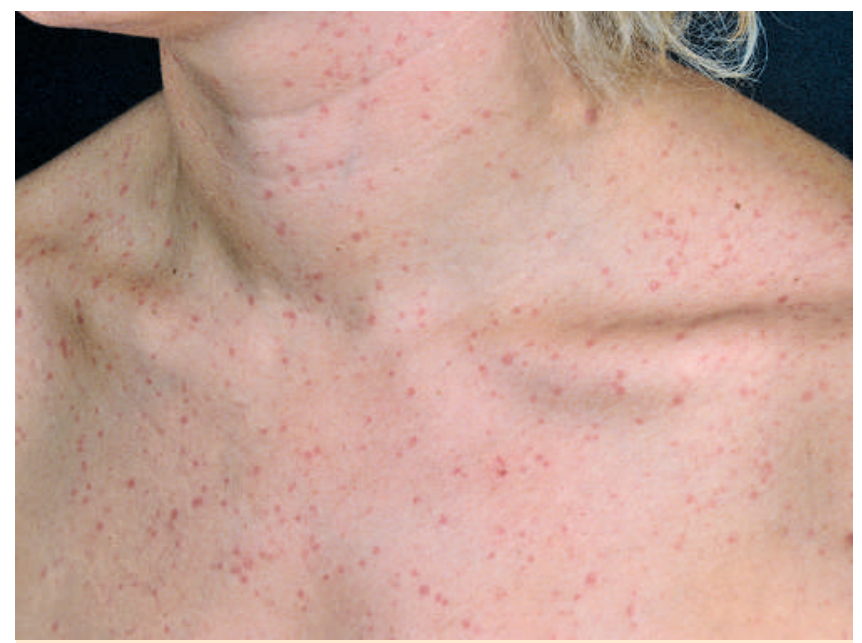

Abb. 450 Jahre alte Patientin, akuter Schub, zahlreiche kleine rote Flecke.

\section{Diagnose}

\section{$\nabla$}

Entscheidend für die Diagnose ist das klinische Bild. Bei einem entsprechenden Verdacht ist es hilfreich, Effloreszenzen zu markieren und den zeitlichen Verlauf zu verfolgen. Die Hauterscheinungen sind flüchtig, mit einer Bestandsdauer von bis zu etwa zwei Stunden. Wenn der Patient meint, der Ausschlag sei zurzeit nicht da, lohnt es sich trotzdem nachzuschauen: Einige Effloreszenzen sind fast immer vorhanden. Juckreiz ist meist gering, z.T. fehlt er auch. In der Allergieambulanz der Stuttgarter Hautklinik wird das Krankheitsbild seit langem etwa einmal pro Quartal diagnostiziert. Anfangs erfolgten Provokationstests zum Ausschluss einer cholinergischen Urtikaria. Später erschien das nicht mehr erforderlich: Die adrenerge Urtikaria weist die beschriebenen krankheitstypischen Hauterscheinungen auf, sie bilden sozusagen das Negativbild zur cholinergischen Urtikaria. Hingewiesen sei auf Folgendes: Bei den anfänglichen Schwitztests traten gelegentlich situationsbedingt vermehrt Effloreszenzen der adrenergen Urtikaria auf, allerdings kein eigentlicher Schub. Vor allem sind es dann nicht die Effloreszenzen der cholinergischen Urtikaria. 


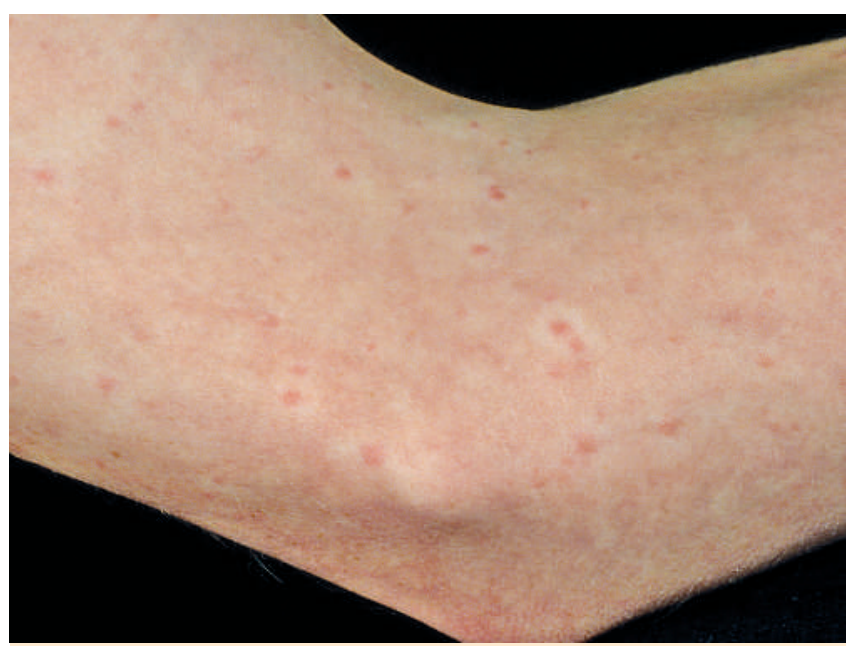

Abb. 521 Jahre alter Patient, typische Effloreszenzen an einem Arm.

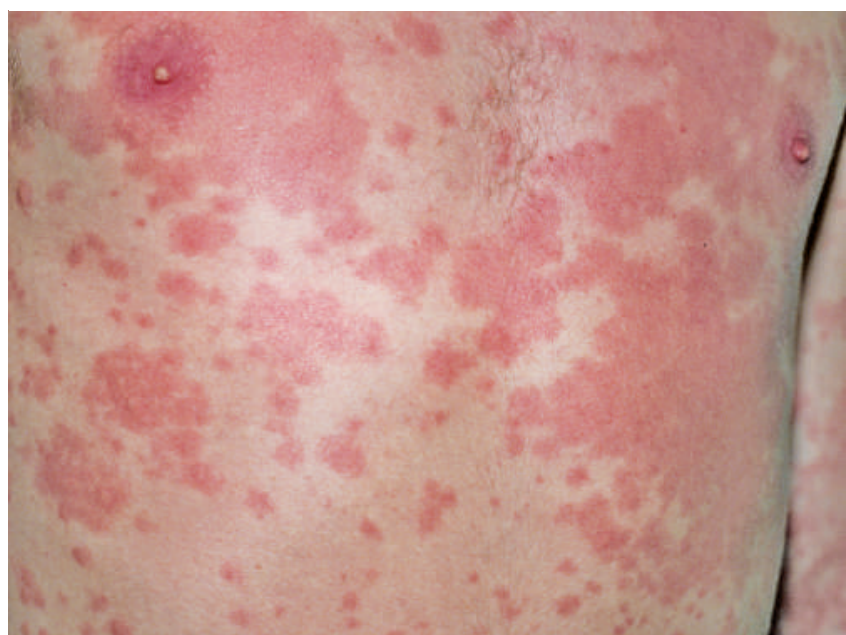

Abb. 6 Gleicher Patient wie in $\oslash$ Abb. 5, makulös-urtikarielles Exanthem 45 Minuten nach $100 \mathrm{mg}$ Azetylsalizylsäure.

\section{Therapie \\ $\nabla$}

Eine wirklich wirksame medikamentöse Therapie existiert nicht. Wichtig ist es, den Patienten dahingehend aufzuklären, dass die Hautveränderungen harmlos sind; dass sie wahrscheinlich durch Anspannung, Aufregung verursacht sind. Der Begriff „Stress“ oder „Stress-Urtikaria“ ist durchaus hilfreich. Wird ein belästigender Juckreiz angegeben, kann man ein Antihistaminikum verordnen. Shelley u. Shelley [1] sahen die Therapie mit dem Betablocker Propranolol in ihren zwei Fällen als wirksam an: In einem Fall konnten die Hautveränderungen „nahezu völlig verhindert“ werden, im zweiten Fall eine „Reduktion der Heftigkeit und Häufigkeit der Attacken“ erreicht werden. In eigenen Fällen war das Propranolol nicht in der Weise wirksam, dass die adrenerge Urtikaria darunter deutlich schwächer oder nicht mehr auftrat. Eine geringe Besserung ist natürlich schon dadurch denkbar, dass die Patienten unter einem derartigen peripheren Betablocker die subjektiven Symptome von Stress oder Angst deutlich gemildert empfinden.

\section{Analgetika-Intoleranz \\ $\nabla$}

Patienten mit adrenerger Urtikaria sind in der Regel ASS-intolerant in der Weise, dass sie mit einem akuten urtikariellen oder makulös-urtikariellen Exanthem reagieren. Anfangs wurden die eigenen Patienten regelmäßig mit Azetylsalizylsäure provoziert, nachdem einige in der Vorgeschichte angegeben hatten, auf Azetylsalizylsäure oder Antirheumatika einen Hautausschlag entwickelt zu haben. Wegen der praktisch immer gesehenen positiven urtikariellen Reaktionen wurde schließlich routinemäßig nur das Paracetamol als Ausweichanalgetikum provoziert, mit guter Verträglichkeit. Fallbezogen können natürlich weitere Analgetika im Expositionstest geprüft werden, wenn sich das als notwendig erweist. Manche Patienten gaben an, der jetzige Ausschlag, d.h. die adrenerge Urtikaria, habe sich im Anschluss an eine ASS-Reaktion entwickelt. Gelegentlich stößt man auf die adrenerge Urtikaria als Nebenbefund, wenn man einen Patienten wegen einer anamnestischen Analgetika-Unverträglichkeit berät bzw. untersucht.

\section{Ätiopathogenese \\ $\nabla$}

Emotionaler Stress ist sicher auslösend, auf jeden Fall für die akuten Schübe. Shelley u. Shelley [1] sahen die Katecholamine Adrenalin und Noradrenalin im Serum ihrer Patienten erhöht. Diese Befunde konnten bei früheren eigenen Untersuchungen bestätigt werden [6]. Shelley u. Shelley [1] stellten weiter die Theorie auf, Noradrenalin könne direkt die Effloreszenzen in der Haut auslösen. Sie sahen bei der intrakutanen Injektion von Noradrenalin eine kleine zentrale Rötung, mit einem hellen Hof; also eine Effloreszenz ähnlich der der adrenergen Urtikaria. Dieses Phänomen ließ sich bei eigenen Patienten nur angedeutet auslösen; allerdings auch bei Vergleichspersonen. Es erscheint schwer vorstellbar, dass Noradrenalin oder Adrenalin die Effloreszenzen der adrenergen Urtikaria in der Haut direkt auslösen können.

Ursächlich für die Hauterscheinungen der adrenergen Urtikaria könnten eher stressbegleitende Transmitter oder Mediatoren sein. Die Hauterscheinungen könnten z.B. durch Neuropeptide ausgelöst werden. Wenn man die ASS-Empfindlichkeit der Patienten mit einbezieht, könnten Leukotriene eine Rolle spielen. Im Hinblick auf die Leukotriene ist es interessant, dass die adrenerge Urtikaria auch bei ASS-empfindlichen Asthmatikern auftritt. Die pathophysiologischen Vorgänge bei der adrenergen Urtikaria sind letztlich nicht geklärt.

\section{Abstract}

\section{Adrenergic Urticaria \\ $\nabla$}

Adrenergic urticaria seems to be more common than the very few reported cases might suggest. In this review the clinical features of adrenergic urticaria are shown in five cases. Emotional stress provokes acute attacks, but mostly few lesions are also seen in intervalls. Intolerance towards acetylsalicylic acid seems to be common with adrenergic urticaria patients. The pathologic mechanisms are not clear, stress-related neuropeptides or leukotrienes may be important. 


\section{Literatur}

1 Shelley WB, Shelley ED. Adrenergic urticaria: a new form of stress-induced hives. Lancet 1985; 2: 1031 - 1033

2 Haustein UF. Adrenergic Urticaria and Adrenergic Pruritus. Acta Derm Venereol 1990; 70: 82-84

3 Figueiredo A, Goncalo M, Paiva I, Poiares-Baptista A. Adrenergic urticaria. Diabetes Care 1988; 11: 440- 441
4 Vithayasai P, Vithaysai V. Adrenergic urticaria: a first report from Thailand. J Med Assoc Thai 1989; 72: 478-480

5 Maerens-Tchokokam B, Vigan M, Breuillard F, Vuitton DA, Girardin P, Laurent R. Guess what: adrenergic urticaria. Eur J Dermatol 1999; 9: $137-138$

6 Kleinhans D, Finkbeiner H. Adrenerge Urtikaria. Z Hautkr 1994; 69: 334-336

\section{Buchbesprechung}

Lasertherapie in der Dermatologie - Atlas und Lehrbuch M. Landthaler, U. Hohenleutner

Heidelberg: Springer, 2006. 2. vollst. überarb. Aufl., 212 S.,

175 Farbabb. Geb. 119,95€

ISBN 3-540-30091-0

Die Entwicklung der Lasermedizin ist rasant, somit erschien eine komplett überarbeitete Neuauflage von Landthalers und Hohenleutners „Lasertherapie in der Dermatologie“ nach sieben Jahren notwendig. Der Inhalt ist dem neuesten Stand der Medizin angepasst, das Konzept gut und bewährt. Auf 212 Seiten erfährt der Leser alles Erforderliche über die biophysikalischen Grundlagen der Lasermedizin, die klinischen Anwendungsgebiete und Wissenswertes zum Thema Lasersicherheit. Die physikalischen Grundlagen werden gut verständlich erklärt, Schemazeichnungen verdeutlichen physikalische Zusammenhänge. Technische Begriffe wie Güteschaltung oder physikalische Vorgänge wie selektive Photothermolyse sind medizinerverständlich dargestellt. Abgerundet wird der Einführungsteil mit einer übersichtlichen Darstellung der in der Dermatologie verwendeten Laser und ihrer Einsatzgebiete.

Den Hauptteil des Buches machen die klinischen Anwendungen aus. In zwölf Kapiteln werden die Einsatzgebiete der Laser von vaskulären Fehlbildungen über maligne Tumoren bis zu Epilation und photodynamischer Therapie eingehend beleuchtet. Für Einsteiger und auch Erfahrene sehr nützlich sind die klaren Empfehlungen der Autoren zur Therapie des jeweiligen Krankheitsbildes. Sie geben Aussagen zum zu verwendenden Laser, zu Therapieregimen und Alternativen. Im Text finden sich häufig auch Angaben zur weiteren Einstellung des Lasers wie zum Beispiel notwendige Energiedosis oder Pulsdauer. Die Behandlungsergebnisse werden durch 175 mehrfarbige Abbildungen im Vorher-Nachher-Stil dokumentiert.
Der kurzgefasste letzte Teil geht auf die gesetzlichen Bestimmungen zu Lasersicherheit und Anwendungen am Menschen sowie auf die notwendigen Schutzmaßnahmen ein.

Das Buch ist klar strukturiert und gut gegliedert. Das Layout ist übersichtlich, teils farblich gegliedert. Der Stil ist gut verständlich, bei Behandlungsempfehlungen kurz und prägnant, teilweise sogar im Telegrammstil. Die Abbildungen sind durchweg mehrfarbig, die Druckqualität sehr gut.

Landthaler und Hohenleutner präsentieren kondensiert das notwendige Wissen zur Lasermedizin in der Dermatologie, hinterlegt mit einer beeindruckenden Bandbreite klinischer Fallbeispiele. Das Konzept mit einem Mix aus medizinischer Information und praktischen Anwendungstipps lässt das Buch für Einsteiger zum „Kochbuch“, für Erfahrene zum Nachschlagewerk werden. Es wird seinem Untertitel „Atlas und Lehrbuch“ absolut gerecht. Dies Buch ist sicherlich sein Geld wert, obwohl 120 Euro für gut zweihundert Seiten nicht gerade wenig erscheinen. Gute Abbildungsqualität mehrfarbig auf hochwertigem Papier und ein überschaubarer Abnehmerkreis fordern ihren Tribut. Die Neuauflage von „Lasertherapie in der Dermatologie" ist eine klare Kaufempfehlung für die auf diesem Gebiet tätigen Kollegen, es bietet das notwendige Wissen verbunden mit klaren Behandlungsempfehlungen auf dem aktuellen Stand der Medizin.

W. Koenen, Heidelberg 